\title{
Selected papers from the third European Conference on Microfluidics: $\mu$ Flu'12
}

\author{
Stéphane Colin · Gian Luca Morini • \\ Juergen J. Brandner
}

Published online: 13 June 2014

(c) Springer-Verlag Berlin Heidelberg 2014

The third European Conference on Microfluidics ( $\mu$ Flu' 12) was held in Heidelberg in December 2012, under the sponsorship of the Hydrotechnic Society of France (SHF), the Alma Mater Studiorum University of Bologna, the University of Toulouse and the Karlsruhe Institute of Technology. This event has gathered 252 participants from 33 countries. During 3 days, 174 communications have been presented in 16 thematic sessions including Liquid Microflows, Gas Microflows, Microsensors and In-Situ Analytics, Fluidic Microactuators and Micromixing, Microfabrication Techniques for Microfluidic Systems, Convective Micro Heat Transfer, Micro Chemical Engineering, Lab-on-a-Chip, Electrokinetic Microflows, Microdroplets Management, Microflows in Biological Systems and Bioengineering, Multi-Phase Flows in Microsystems, Microflow Visualization, and other topics. Two special sessions have been devoted to Industrial Applications of Microfluidics and to Nanofluidics.

The multi-disciplinarity of Microfluidics is highlighted by the variety of works presented in this Conference which

\section{S. Colin}

INSA, Institut Clément Ader (ICA), Université de Toulouse,

135, Avenue de Rangueil, 31077 Toulouse, France

e-mail: stephane.colin@insa-toulouse.fr

G. L. Morini $(\square)$

DIN, Alma Mater Studiorum Università di Bologna,

40136 Bologna, Italy

e-mail: gianluca.morini3@unibo.it

\section{J. J. Brandner}

Institute for Micro Process Engineering (IMVT),

Karlsruhe Institute of Technology, Campus North,

Hermann-von-Helmholtz-Platz 1,

76344 Eggenstein-Leopoldshafen, Germany

e-mail: juergen.brandner@kit.edu confirms also that Microfluidics nowadays finds applications in every industrial sector like biology, medicine, chemical and process engineering, transports, environment, microelectronics.

This year the Scientific Committee has selected four papers presented to the Conference for publication in $M i$ crofluidics and Nanofluidics; the common topic of these selected papers is the detailed analysis of the interactions at micrometric scale between the fluid and the solid walls of a microdevice by highlighting what happens when the solid surface either presents microgrooves, or gathers electrical charges, or has been submitted to a surface chemical treatment or is simply oscillating.

More in detail, the paper of Gopalan and Kandlikar is devoted to the experimental analysis of the wettability of micro-patterned surfaces. Their results show that the wettability is strongly influenced by the spacing factor of a grooved surface, defined as the ratio between the channel depth and the channel width: The spacing factor influences the contact angle, the contact angle hysteresis, and the transition characteristics between the Cassie and Wenzel states. The authors demonstrate that their experimental results can be useful for the design of the gas channels of a Proton Exchange Membrane Fuel Cell (PEMFC) in order to optimize the water drainage.

The paper presented by Gentili et al. is focused on the analysis of the combined effect of nanoscale roughness and surface chemistry modification in order to achieve large liquid slippage due to entrapment of air pockets in the surface asperities. In the paper, a numerical simulation campaign based on full atoms Molecular Dynamics aimed at reproducing a realistic superhydrophobic system comprising water, solid walls, and hydrophobic coating with octadecyltrichlorosilane (OTS) is described and compared with the results obtained by following a continuum 
approach. The numerical results presented in this paper highlight how pressure influences the shape of the liquid/ vapor interface as well as the complex interplay between water and hydrophobic chains close to the triple line where it significantly alters the liquid structure, thus modifying apparent slippage. This effect is an intrinsically atomistic phenomenon that cannot be predicted at the continuum level.

The paper of Liu et al. presents an experimental analysis of the vortical structures generated in AC electroosmotic (ACEO) flows using astigmatism micro-Particle-Tracking Velocimetry (astigmatism $\mu$-PTV). The experimental velocity field is used to validate an extended nonlinear Gouy-Chapman-Stern (GCS) model accounting for the surface conduction effect. In the paper, the primary fluid circulation, given in terms of the spanwise component of vorticity, is numerically and experimentally analyzed as a function of frequency and amplitude of the AC voltage.

The paper presented by Tsuji and Aoki is aimed at investigating, by means of a kinetic-theoretic approach, unsteady motion of a gas between two parallel plates in the case where one of the plates starts (harmonic) oscillation in its normal direction. The Bhatnagar-GrossKrook (BGK) model of the Boltzmann equation is solved numerically for wide ranges of operative parameters, such as the Knudsen number and the Mach number, with special interest in the fully nonlinear wave motion.

Our sincere thanks to all the authors for presenting their works at the Conference and in this special issue. We would also like to extend our thanks to all the members of the Scientific Committee of the Conference for the accurate review process of each paper before the presentation to the Conference and, after the Conference, for the selection of the papers for the special issues linked to the Conference. Our special thanks to Prof. Roland Zengerle for his foresight in dedicating this volume to the Conference. We invite all the readers of Microfluidics and Nanofluidics to participate to the fourth European Conference on Microfluidics ( $\mu$ Flu'14) in Limerick, Ireland, in December 2014. Further information on the Conference is available at http:// www.microfluidics2014.eu. 